\title{
Cardiovascular transition at birth: a physiological sequence
}

\author{
Stuart B. Hooper ${ }^{1,2}$, Arjan B. te Pas ${ }^{3}$, Justin Lang ${ }^{1}$, Jeroen J. van Vonderen ${ }^{3}$, Charles Christoph Roehr ${ }^{1,4}$, Martin Kluckow ${ }^{5}$, \\ Andrew W. Gill ${ }^{6}$, Euan M. Wallace ${ }^{1,2}$ and Graeme R. Polglase ${ }^{1,2}$
}

The transition to newborn life at birth involves major cardiovascular changes that are triggered by lung aeration. These include a large increase in pulmonary blood flow (PBF), which is required for pulmonary gas exchange and to replace umbilical venous return as the source of preload for the left heart. Clamping the umbilical cord before PBF increases reduces venous return and preload for the left heart and thereby reduces cardiac output. Thus, if ventilation onset is delayed following cord clamping, the infant is at risk of superimposing an ischemic insult, due to low cardiac output, on top of an asphyxic insult. Much debate has centered on the timing of cord clamping at birth, focusing mainly on the potential for a time-dependent placental to infant blood transfusion. This has prompted recommendations for delayed cord clamping for a set time after birth in infants not requiring resuscitation. However, recent evidence indicates that ventilation onset before cord clamping mitigates the adverse cardiovascular consequences caused by immediate cord clamping. This indicates that the timing of cord clamping should be based on the infant's physiology rather than an arbitrary period of time and that delayed cord clamping may be of greatest benefit to apneic infants.

$\mathbf{T}$ he transition from fetal to newborn life at birth represents a major physiological challenge that all humans must undertake to survive. Before birth the future airways of the lungs are liquid-filled and the lungs take no part in gas exchange, which instead occurs across the placenta (1). In addition, pulmonary blood flow (PBF) is low because pulmonary vascular resistance (PVR) is high, redirecting the majority of right ventricular output (RVO) through the ductus arteriosus (DA) and into the systemic circulation $(2,3)$. While this physiological arrangement provides the fetus with key adaptive advantages that allow it to survive and flourish in utero, they are not conducive to survival after birth. In particular, separating the infant from the placenta (the fetal organ of gas exchange) by clamping the umbilical cord necessitates the rapid switch to pulmonary gas exchange within minutes of birth. This switch not only involves aeration of the airways and gas-exchange regions of the lung, but also includes a major reorganization of the fetal cardiovascular system (2-4). Specifically, PVR must decrease rapidly so that $\mathrm{PBF}$ can increase and become the sole recipient of RVO. This is not only critical for ensuring that the lung's gas-exchange efficiency is adequate, but also critical for increasing PBF and enabling it to become the sole source of preload for the left ventricle $(5,6)$. This is because PBF is the only source of venous return for the left heart in adults and so PBF must have the capacity to replace umbilical venous return as the primary source of preload for the left ventricle when the cord is clamped. As all of these events must occur within minutes of birth and all are critical for survival, it is not surprising that they are linked and mostly triggered by a single event: lung aeration $(5,6)$. Lung aeration triggers the decrease in PVR and increase in PBF, which, combined with umbilical cord clamping, initiates a sequence of changes that dramatically reorganize the infant's circulation. As a result, major vascular shunts close, leading to anatomical separation of the pulmonary and systemic circulations and right and left sides of the heart, transforming the newborn's circulation from a fetal circuit into the adult phenotype (2).

Such is the importance of these changes that much research has focused on the circulatory transition at birth. However, until recently the inter-relationships between lung aeration, the increase in PBF, umbilical cord clamping, and flow reversal through the DA have not been examined (5). In particular, the changes have not been recognized as an integrated sequence of events that are highly interdependent and may significantly impact on the infant's cardiovascular function in the newborn period. This review will focus on recent developments that have advanced our understanding of how the infant transitions at birth and have provided new insights into improving clinical practice, such as the appropriate timing for umbilical cord clamping.

\section{LUNG AERATION “TRIGGERS"THE INCREASE IN PULMONARY BLOOD FLOW AT BIRTH}

By maintaining the lungs in a distended state, the presence of liquid within the future airways during fetal life plays an essential role in lung development (1). However, at birth the

\footnotetext{
${ }^{1}$ The Ritchie Centre, MIMR-PHI Institute of Medical Research, Monash University, Clayton, Victoria, Australia; ${ }^{2}$ Department of Obstetrics and Gynecology, Monash University, Clayton, Victoria, Australia; ${ }^{3}$ Department of Pediatrics, Leiden University Medical Centre, Leiden, The Netherlands; ${ }^{4}$ Monash Newborn, Monash Medical Centre, Clayton, Victoria, Australia; ${ }^{5}$ Department of Neonatalogy, Royal North Shore Hospital and University of Sydney, Sydney, New South Wales, Australia; ${ }^{6} \mathrm{Centre}$ for Neonatal Research and Education, The University of Western Australia, Perth, Western Australia, Australia. Correspondence: Stuart B. Hooper (stuart.hooper@monash.edu) 


\section{Circulatory changes at birth $\mid$ Review}

airways must be cleared of this liquid to allow the entry of air and the onset of pulmonary gas exchange. Recent X-ray imaging studies have shown that after birth, airway liquid clearance predominantly occurs due to inspiratory activity. This creates a hydrostatic pressure gradient that facilitates the movement of liquid from the airways into the surrounding tissue (7-9). As a result, liquid accumulates within the interstitial tissue compartment forming perivascular fluid cuffs (10), which increases pressure within the lung's interstitial tissue compartment (11). As liquid is cleared from the airways (typically within the first 3-5 breaths) much more quickly than from lung tissue (about $4 \mathrm{~h}$ ), the chest wall must expand to accommodate the increase in lung volume caused by the increase in resting lung air volume $(5,7,12)$. This highlights the importance of the infant having a compliant chest wall at birth during the transition. In the absence of a compliant chest wall, failure of the chest wall to expand would increase intrathoracic pressures and interfere with the infant's respiratory and cardiovascular systems, the latter through reducing venous return and increasing PVR.

Although lung aeration is accepted as the primary trigger for the increase in PBF at birth (Figure 1) (4), what remain less certain are the mechanisms by which aeration exerts this effect. A number of mechanisms are likely to be involved including increased oxygenation, enhanced activity of vasodilator agents, and a variety of mechanical effects associated with lung aeration $(3,13)$. These include a "mechanical effect" of ventilation and changes in alveolar/capillary transmural pressures resulting from the formation of surface tension, which increases lung recoil despite the presence of surfactant $(14,15)$. These topics have been covered in an excellent recent review (3) and so will not be discussed in detail here. Instead, we will focus on more recent insights, which suggest that a key component of the sequence of events leading to the decrease in PVR and increase in PBF at birth may have gone largely unnoticed.
The rationale underpinning all of the proposed mechanisms responsible for the increase in $\mathrm{PBF}$ at birth suggests that the response is solely activated by air entry (4), which presumably initiates vasodilation in aerated regions in a locally dependent manner. This assumption is based on a number of facts as well as information extrapolated from adult physiology. For instance, increasing and decreasing fetal oxygenation are well known (3) to increase and decrease PBF, respectively, independently of other factors. Similarly, i.v. administration of vasodilators can vasodilate the pulmonary vascular bed and increase $\operatorname{PBF}(3,13)$, although this vasodilation cannot be sustained. While this shows that the perinatal lung is responsive to oxygen and vasodilators, it also indicates that air entry must cause irreversible changes that act to alter the signaling pathways for key vasoactive agents causing localized vasodilation (3). The latter is an extrapolation from the known ventilation/ perfusion relationships in adult lung whereby increased alveolar oxygen levels cause localized vasodilation through nitric oxide-mediated pathways (16). This increases blood flow to well-ventilated lung regions ensuring appropriate matching of ventilation and blood flow. In addition, the introduction of air into the lungs causes surface tension to form and increase lung recoil in a locally dependent manner $(15,17)$. The increase in alveolar wall recoil increases capillary/alveolar wall transmural pressures, which increases capillary caliber in aerated regions (4). Alterations in alveolar/capillary wall transmural pressures are a well-established determinant of pulmonary capillary flow and are a major determinant of vascular resistance within the mature lung $(18,19)$. The latter is responsible for the welldescribed vertical distribution of capillary blood flow within the adult lung and alveolar capillary recruitment that markedly reduces PVR with increasing cardiac output.

Based on this knowledge, the spatial relationship between regional lung aeration and regional changes in PBF has recently been examined with the expectation that regional

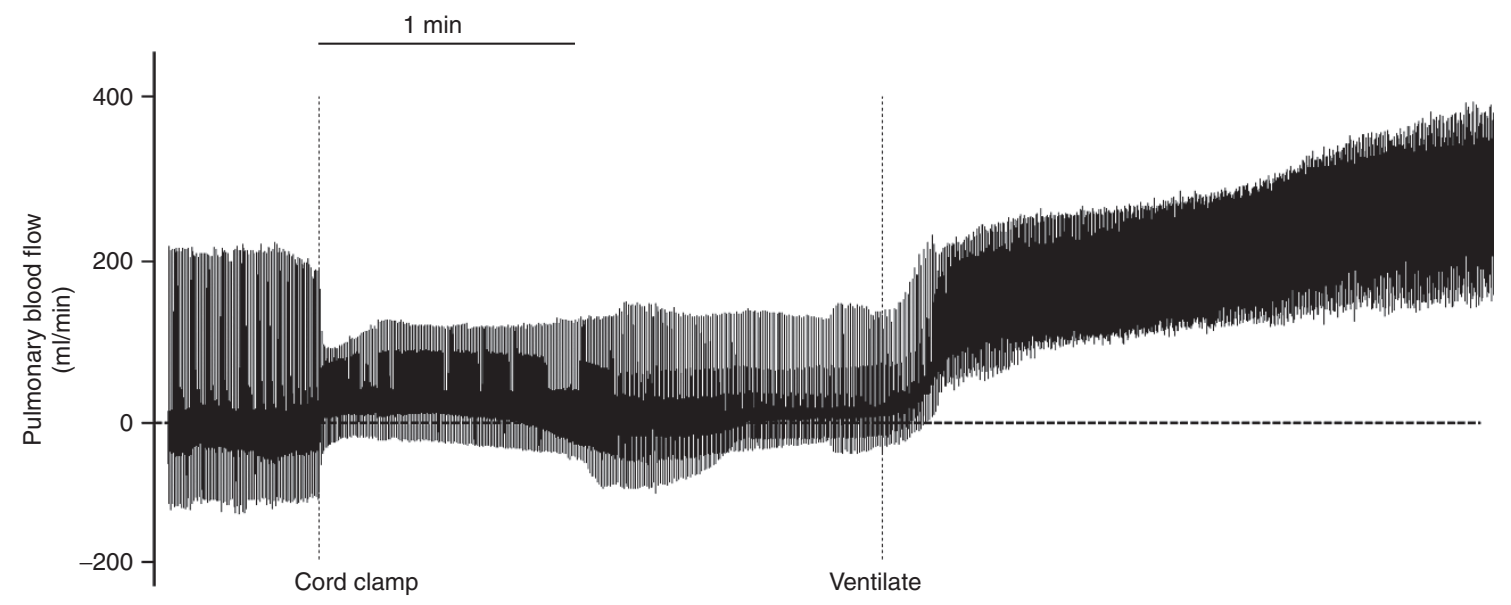

Figure 1. Instantaneous pulmonary blood flow (PBF) measured before, during, and after umbilical cord clamping (cord clamp) and after ventilation onset (ventilate); each spike represents a single heartbeat. Note that before cord clamping, PBF oscillates around zero during the cardiac cycle, with maximum values indicating peak systolic flows and minimum values indicating flows during diastole. Positive PBF values indicate antegrade flow into the lungs whereas negative values indicate retrograde flow away from the lungs. While cord clamping markedly alters the PBF amplitude, which is due to a reduction in blood volume ejected per heartbeat, ventilation onset rapidly and markedly increases PBF, resulting in forward flow into the lungs throughout the cardiac cycle. 


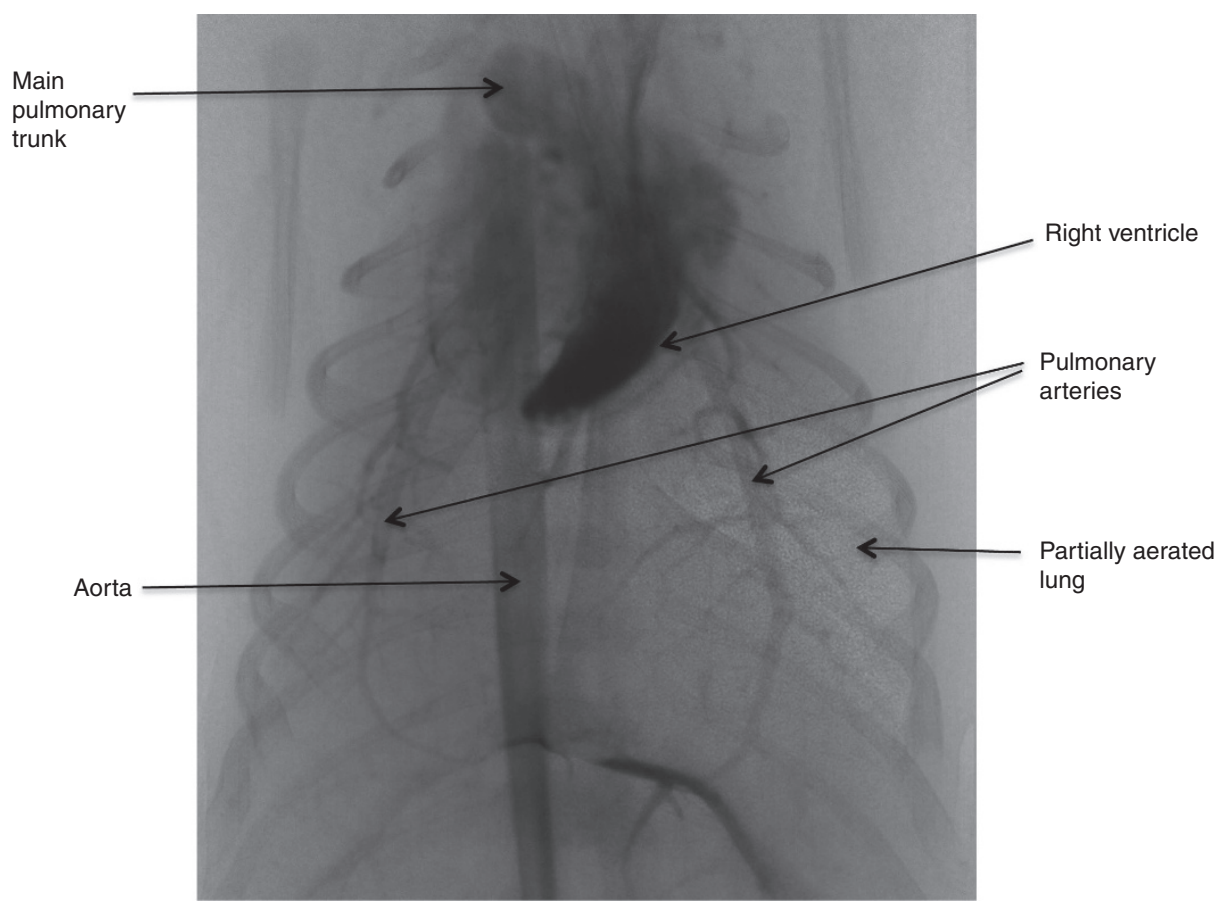

Figure 2. Simultaneous phase contrast and angiographic X-ray image of a near-term rabbit kitten following partial ventilation of the right lung (20). Aerated lung regions can be seen as "speckle" in the image and is due to refraction at the air/water interface (8,34). The pulmonary blood vessels are highlighted by the injection of an iodine contrast agent, which also highlights the right ventricle and the aorta. Despite aeration of only one lung, pulmonary blood flow (PBF) (as indicated by the amount of iodine contrast agent) was observed to increase in both aerated and unaerated lung regions (20).

lung aeration would increase $\mathrm{PBF}$ only in aerated lung regions (20). This was investigated using simultaneous phase contrast $\mathrm{X}$-ray imaging and angiography, which uses the power of imaging to identify the spatial relationships between aeration and blood flow (Figure 2). Contrary to what was expected, regional lung aeration triggered a global increase in PBF, indicating that the increase in PBF was not spatially related to lung aeration (20). This observation alone indicates that our current understanding for how lung aeration stimulates an increase in PBF requires re-evaluation. Indeed, it is possible that an unknown mechanism, possibly independent of oxygen and the other mechanisms known to regulate regional PBF, triggers the initial increase in PBF (20). Whatever the mechanism(s), it is clear that a major ventilation perfusion mismatch occurs in the lung at birth when only a portion of the lung aerates (Figure 2). This is important because regionalized aeration is a common event when initiating ventilation in very preterm infants. To compensate, the caregiver usually increases the oxygen pressure gradient by increasing the inspired oxygen content. However, it is possible that this "spatial disconnection" between lung aeration and increased PBF at birth has adaptive advantages. This is because the increase in PBF is central to maintaining cardiac output after birth, by becoming the sole source of left ventricular preload (see below). Although optimizing pulmonary oxygen exchange in the immediate newborn period is important, maintaining cardiac function is even more important to avoid ischemia contributing to any hypoxic events. If correct, then it would seem preferable to ensure that the increase in PBF and thus left ventricular preload is not limited by an inability to completely aerate the lung at birth. Clearly, further studies are required to determine how lung aeration triggers an increase in PBF in a spatially independent manner and the relative roles of systemic oxygenation and circulating vasodilators.

\section{CARDIOVASCULAR CHANGES AT BIRTH: EFFECT OF CORD CLAMPING}

In comparison to adults, the fetal circulatory system is considerably more complex. Because PVR is high, the majority of RVO bypasses the lungs and flows through the DA (rightto-left shunting), passing directly into the descending thoracic aorta. Much of this output then passes through the placental circulation for, among other things, oxygenation $(2,3)$. As the placental circulation receives a high proportion of total fetal cardiac output (30-50\% depending on species and gestational age), it is also a large source of venous return and preload for the fetal heart (2). In fetal sheep, at least $50 \%$ of umbilical venous return passes through the ductus venosus and, without significant mixing in the inferior vena cava, passes directly into the left atrium through the foramen ovale (2). As a result, umbilical venous return is the main source of preload for the left ventricle, particularly as fetal PBF is very low and is unable to provide sufficient venous return to maintain left ventricular output (2). The finding of a close inverse relationship between PBF and flow through the foramen ovale (FO) in human fetuses indicates that the provision of left ventricular preload is not static, but is dependent upon a competitive inter-relationship between these two sources of venous return (21). Although it 


\section{Circulatory changes at birth | ReView}

is generally assumed that fetal $\mathrm{PBF}$ is perpetually low, based on early fetal studies (2), we now know that this interpretation is not entirely accurate. Indeed, PBF markedly increases (up to fourfold) during fetal breathing movements (FBMs), which is thought to be due to dynamic and transient (breath-by-breath) increases in the capillary/interstitial tissue transmural pressures, leading to a transient decrease in PVR (22). As such, it is likely that the relative contribution of these two sources of preload (PBF and flow through the FO) will vary depending upon factors such as fetal activity.

In the fetus, the presence of the DA and the high PVR confers a unique characteristic pattern to the PBF waveform $(22,23)$, which can be influenced by fetal activity such as FBMs (22). During systole, blood flows toward the lungs but during late systole and throughout most of diastole, blood flows retrogradely in the pulmonary arteries away from the lungs (Figure 3). This retrograde flow is due to blood reflecting off the highly constricted pulmonary vascular bed and exiting the pulmonary circulation by flowing through the DA and entering the systemic circulation (24). As a result, blood flows continuously in the DA throughout the cardiac cycle with relatively high basal flows during diastole (23). As flow in the main pulmonary trunk, which is only $1-2 \mathrm{~cm}$ upstream of the DA, decreases to zero during diastole (2), the high basal flows in the DA during diastole are entirely attributable to retrograde flow in the left and right pulmonary arteries (Figure 3).

\section{Cardiovascular Changes with Immediate Umbilical Cord Clamping}

At birth, immediate clamping of the umbilical cord reduces venous return to the heart by $30-50 \%$ and instantaneously increases systemic vascular resistance by removing the lowresistance placental circulation from the systemic circuit (25). As a result, arterial pressure rapidly increases by about $30 \%$ within four heartbeats and cardiac output decreases by $30-50 \%$. The latter is due to both the increase in afterload and the decrease in venous return (which decreases preload) (25). In addition, cerebral blood flow initially increases, presumably due to the rapid increase in arterial pressure, but then rapidly decreases again as cardiac output decreases and arterial blood pressures stabilize. Cardiac output then remains low until ventilation commences and PBF increases, which restores venous return and left ventricular preload before restoring cardiac output (Figure 4). The relative timing of cord clamping and lung aeration are therefore critical to a smooth transition and, if out of sequence, may expose the newborn to significant cardiovascular instability leading to complications including hemorrhage.

Due to the combined effect of a decrease in PVR and an increase in systemic vascular resistance caused by umbilical cord clamping, net flow through the DA reverses resulting in net left-to-right shunting across the DA (23). As a result, flow through the DA significantly contributes to the increase in PBF immediately after birth, which causes a major change in the PBF waveform and results in a significant left ventricle, lung, left ventricle short circuit. Retrograde PBF is rapidly abolished (within 5-10 min) and blood flows toward the lungs throughout the entire cardiac cycle with the high basal flow during diastole being entirely due to the contribution of leftto-right shunting through the DA. However, although net flow through the DA is left-to-right, instantaneous flow is mostly bidirectional, with right-to-left flow occurring briefly during
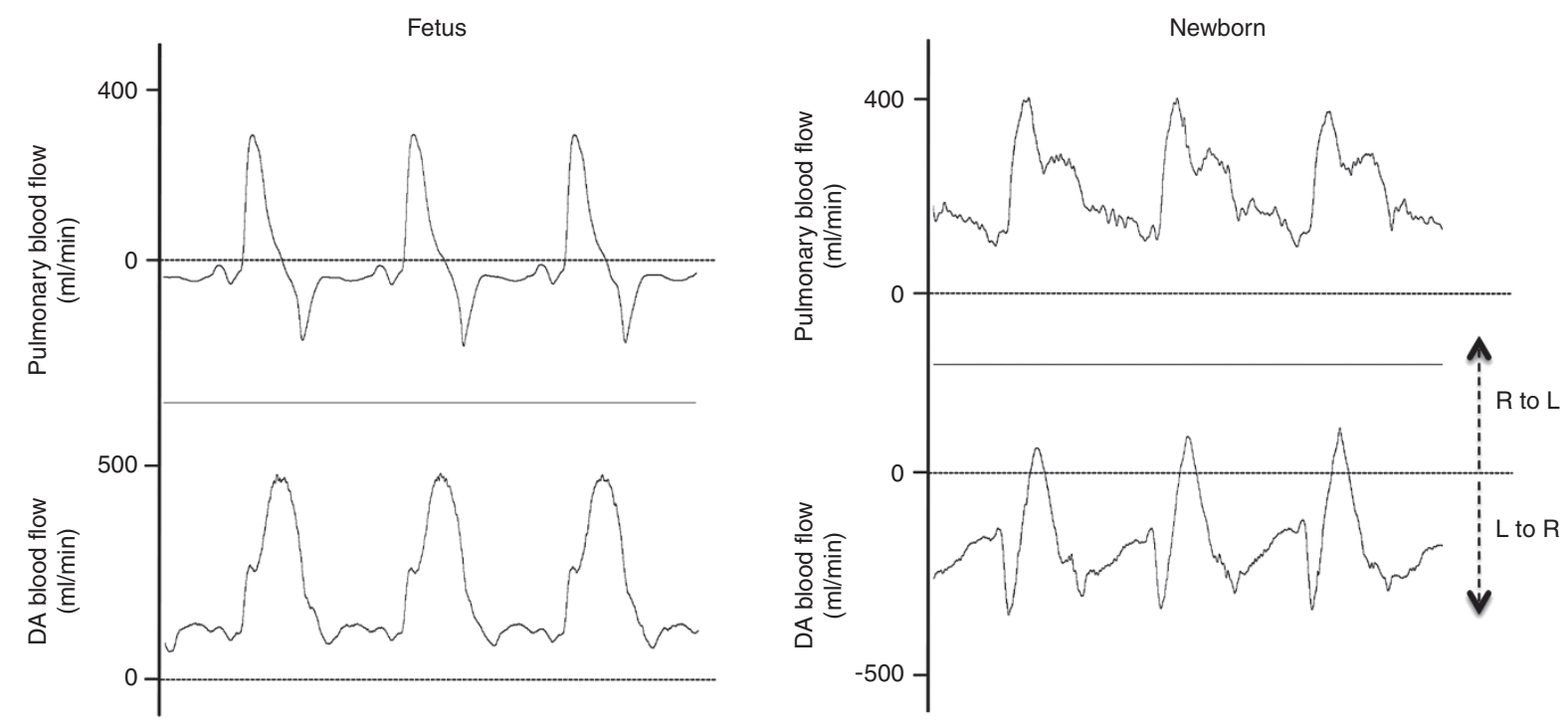

Figure 3. Blood flow waveforms in the left pulmonary artery and ductus arteriosus (DA) throughout three consecutive heartbeats in a lamb before (Fetus) and after (Newborn) ventilation onset. Note the shift in the blood flow waveforms in relation to zero flow before and after ventilation onset. Pulmonary blood flow (PBF) oscillates around zero before ventilation onset (negative flows reflect retrograde flow of blood away from the lungs), but is positive throughout the cardiac cycle after ventilation onset. Before ventilation onset, flow in the DA is positive throughout the cardiac cycle. This indicates that blood flows right-to-left ( $R$ to $L$ ), from the pulmonary circulation into the aorta, continuously throughout the cardiac cycle. $R$ to $L$ DA flow during diastole before ventilation onset is due to retrograde PBF. Following ventilation onset, DA blood flow is predominantly left-to-right ( $L$ to $R$ ), flowing from the aorta and into the lungs, throughout most of the cardiac cycle, except during early systole. L to R flow in the DA following ventilation onset significantly contributes to PBF (23) and is entirely responsible for PBF during diastole. 
Before ventilation onset

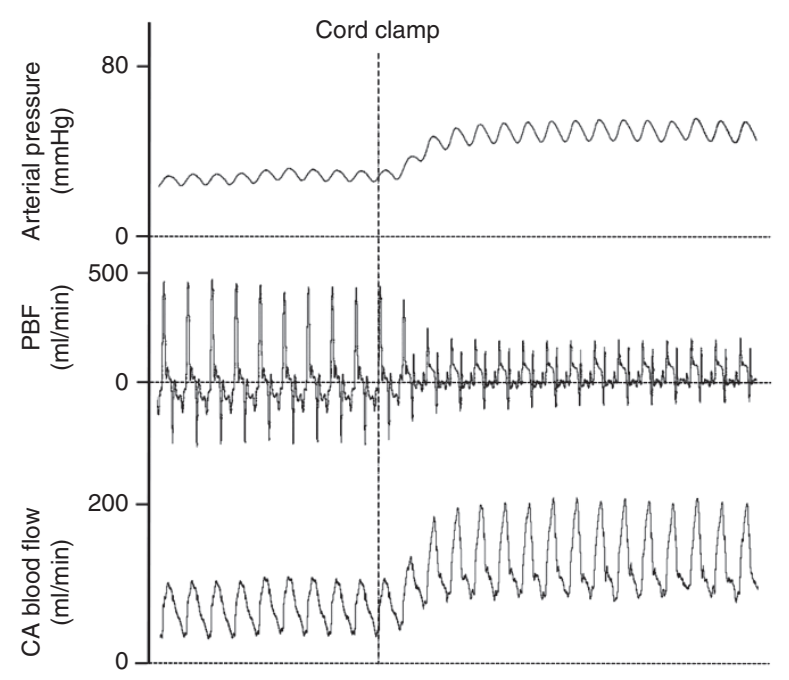

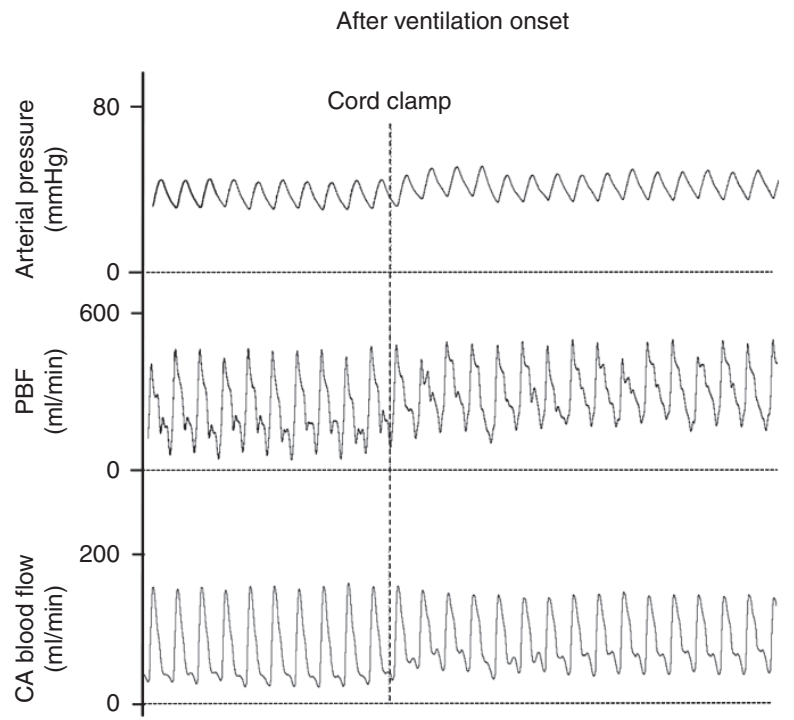

Figure 4. Effect of umbilical cord clamping (cord clamping) on systemic arterial pressure (carotid artery), pulmonary blood flow (PBF), and carotid arterial (CA) blood flow measured in newborn lambs before or after ventilation onset. Note that if cord clamping occurs after ventilation onset, the increases in CA pressure and blood flow are greatly mitigated as is the decrease in right ventricular stroke volume, indicated by maintained amplitude in PBF waveform. The reduced increase in CA pressure is because the pulmonary circulation, due to left-to-right shunting through the ductus arteriosus (DA), can immediately act as an alternate low-resistance pathway for blood flow emanating from the left ventricle (25).

early systole but then switching to left-to-right during late systole and throughout diastole (Figure 3). This is thought to be due to the anatomical relationship between the DA and pulmonary and aortic arteries (5). That is, during systole, the pressure wave exiting the right ventricle reaches the pulmonary artery/DA junction before the pressure wave exiting the left ventricle reaches the DA/aorta junction. As a result, briefly during early systole, the pressure at the pulmonary artery/DA junction is greater than that at the DA/aorta junction resulting in a transient period of right-to-left shunting through the DA (23). However, when the systolic pressure wave from the left ventricle reaches the aortic end of the DA, the pressure gradient reverses resulting in a reversal inflow and left-to-right shunting through the DA.

\section{Cardiovascular Changes with Delayed Umbilical Cord Clamping}

As indicated above, clamping the umbilical cord immediately after birth and before the lungs aerate causes a 30-50\% decrease in cardiac output due to an increase in afterload and a reduction in ventricular preload caused by the loss in umbilical venous return (25). This reduction in cardiac output persists as long as the delay between cord clamping and the increase in PBF (induced by ventilation onset) continues (Figure 4). However, the decrease in ventricular preload can be avoided by increasing the PBF before the umbilical cord is clamped (25). As the increase in PBF results from lung aeration, establishing pulmonary ventilation while the umbilical cord is still intact allows PBF to increase while venous return and ventricular preload are maintained by umbilical venous return (25). As a result, $\mathrm{PBF}$ can immediately take over the role of supplying ventricular preload before the cord is clamped and umbilical venous return is lost. Thus, initiating ventilation before the cord is clamped can mitigate the changes in cardiac output, blood pressure, and cerebral blood flow caused by umbilical cord clamping in preterm lambs (Figure 4) (25).

The appropriate timing of umbilical cord clamping has been a topic of debate for millennia (26), even dating back to Aristotle in 350 BC. The concept that umbilical cord clamping should not occur immediately after birth is often termed "delayed" cord clamping, although this implies that "immediate cord" clamping is the normal or natural timing for cord clamping. This may not be correct. Nonetheless, immediate cord clamping after birth is the most commonly used and accepted practice worldwide and forms part of the current strategy for actively managing the third stage of labor; the rationale for which is to reduce the risk of maternal hemorrhage (27). The active thirdstage management of labor includes oxytocin administration upon delivery of the infant's anterior shoulder and immediate cord clamping (27). While it is established that active management reduces the risk of severe post-partum hemorrhage $(\mathrm{PPH})$, it is focused on maternal outcomes and may overlook potential adverse affects on the infant. Indeed, one adverse finding detailed in the Cochrane review showed that maternal oxytocin administration caused a significant decrease in birth weight (27), presumably due to a reduction in neonatal blood volume. As all infants had immediate cord clamping (27), this finding is consistent with the view that delayed cord clamping causes placental to infant blood transfusion. There is also evidence that oxytocin administration after delivery of the placenta is associated with less maternal blood loss than administration before delivery of the placenta (28) and that early administration of oxytocin may increase the risk of a retained placenta (29). Perhaps, therefore, it is time to revisit how the third stage of labor is managed with reference to the timing of oxytocin administration and umbilical cord clamping so that both maternal and neonatal outcomes are considered. 


\section{Circulatory changes at birth $\quad$ Review}

The available evidence, derived from both clinical and experimental studies, indicates that the timing of umbilical cord clamping can have significant consequences for the infant, with most attention focusing on the potential for placental to infant blood transfusion $(30,31)$. Indeed, it is possible that an increase in infant blood volume can account for some of the cardiovascular benefits detailed above. As a result, the most recent (2010) guidelines from the International Liaison Committee for Resuscitation (ILCOR) have recommended that umbilical cord clamping be delayed for at least $1 \mathrm{~min}$ in healthy term infants not requiring intervention (32). However, the optimal timing for the delay in cord clamping is not clear. In view of recent evidence, clamping the cord at a set arbitrary period of time after birth with no reference to the infant's changing physiology would not appear to be physiologically sound or likely to optimize the potential benefits for an individual $(25,26)$. The most recent experimental evidence indicates that, rather than an arbitrary period of time, the infant's respiratory function is likely to be a better indicator for when the cord should be clamped (25). That is, waiting until the infant has established effective breathing, particularly if a pulse oximeter is used and showing an increasing oxygen saturation level, will ensure that PBF has increased and is able to provide sufficient venous return and preload for the left ventricle. Most infants will start breathing and crying immediately after birth and, therefore, may receive little benefit from a 1-min delay in cord clamping, unless it receives extra blood volume. On the other hand, those infants who experience a delay in establishing breathing after birth may receive substantial benefit from delayed cord clamping (25), particularly if it allows time for spontaneous breathing to commence. In any event, the mother may also receive benefit from delayed cord clamping and administration of oxytocin after placental delivery (26).

In reference to the 2010 ILCOR guidelines, delayed umbilical cord clamping is only recommended for healthy term infants not requiring intervention (32). It has also been suggested that delayed cord clamping is contraindicated for neonatal asphyxia and, instead, it is recommended that the infant should be immediately separated from the placenta and transferred for urgent resuscitation (33). However, it can be argued that delayed cord clamping would be of least benefit to infants not requiring intervention and of most benefit to those requiring respiratory support immediately after birth. Indeed, volume administration is an important first step in the care of sick infants. But it is also important to recognize that the benefits of delayed cord clamping in apneic asphyxic infants will likely depend on the cause of the asphyxia. If it is due to cord compression or a placental complication, then it is unlikely that delaying cord clamping until after ventilation onset will have any benefit. Clearly, further studies are required to define whether infants requiring resuscitation at birth, particularly preterm and asphyxic infants, would be better served by receiving that support while still attached to the umbilical cord.

\section{SUMMARY}

It is now well established that lung aeration, the increase in PBF, and maintenance of cardiac function in the newborn period are intimately linked (5). More specifically, these major physiological events occur in a set interdependent sequence, beginning with lung aeration, which triggers an increase in PBF that then provides venous return and preload for the left ventricle (5). As left ventricular preload in the fetus is predominantly derived from umbilical venous return (2), if the period between umbilical cord clamping and breathing onset is delayed at birth, the infant will experience a period of reduced cardiac function. This is due to a reduction in ventricular preload caused by the loss of umbilical venous return and the inability of pulmonary venous return to supply sufficient preload before the lung aerates and PBF increases (25). If this is combined with a transient period of hypoxia, the infant is at risk of experiencing a hypoxic/ischemic event. Alternatively, if ventilation commences before the cord is clamped, there is no loss in preload as venous return immediately switches from umbilical to pulmonary venous return upon cord clamping. In view of this improved understanding of the inter-relationships between these major physiological events that underpin the transition to newborn life, the logic for delaying cord clamping until after breathing has begun is now more readily apparent.

\section{STATEMENT OF FINANCIAL SUPPORT}

This research was supported by National Health and Medical Research Council (Australia) Program Grant (606789) and Research Fellowships (GRP: 1026890 and SBH: 545921), a Rebecca L. Cooper Medical Research Foundation (Australia) Fellowship (GRP), the Eunice Kennedy Shriver National Institute of Child Health \& Human Development of the National Institutes of Health (Bethesda, MD, USA; Award Number R01HD072848), and a Financial Markets Foundation for Children (Australia) grant as well as the Victorian government's Operational Infrastructure Support Program (Australia).

Disclosure: The authors do not have any financial ties to products in the study or potential/perceived conflicts of interest to declare.

\section{REFERENCES}

1. Hooper SB, Harding R. Fetal lung liquid: a major determinant of the growth and functional development of the fetal lung. Clin Exp Pharmacol Physiol 1995;22:235-47.

2. Rudolph AM. Fetal and neonatal pulmonary circulation. Annu Rev Physiol 1979;41:383-95.

3. Gao Y, Raj JU. Regulation of the pulmonary circulation in the fetus and newborn. Physiol Rev 2010;90:1291-335.

4. Hooper SB, Harding R. Role of aeration in the physiological adaptation of the lung to air-breathing at birth. Curr Respir Med Rev 2005;1:185-95.

5. Hooper SB, Siew ML, Kitchen MJ, te Pas AB. Establishing functional residual capacity in the non-breathing infant. Semin Fetal Neonatal Med 2013;18:336-43.

6. te Pas AB, Davis PG, Hooper SB, Morley CJ. From liquid to air: breathing after birth. J Pediatr 2008;152:607-11.

7. Hooper SB, Kitchen MJ, Wallace MJ, et al. Imaging lung aeration and lung liquid clearance at birth. FASEB J 2007;21:3329-37.

8. Kitchen MJ, Lewis RA, Morgan MJ, et al. Dynamic measures of regional lung air volume using phase contrast $\mathrm{x}$-ray imaging. Phys Med Biol 2008;53:6065-77.

9. Lewis RA, Yagi N, Kitchen MJ, et al. Dynamic imaging of the lungs using X-ray phase contrast. Phys Med Biol 2005;50:5031-40. 
10. Bland RD, McMillan DD, Bressack MA, Dong L. Clearance of liquid from lungs of newborn rabbits. J Appl Physiol Respir Environ Exerc Physiol 1980;49:171-7.

11. Miserocchi G, Poskurica BH, Del Fabbro M. Pulmonary interstitial pressure in anesthetized paralyzed newborn rabbits. J Appl Physiol (1985) 1994;77:2260-8.

12. Hooper SB, te Pas AB, Lewis RA, Morley CJ. Establishing functional residual capacity at birth. NeoReviews 2010;11:474-83.

13. Fineman JR, Soifer SJ, Heymann MA. Regulation of pulmonary vascular tone in the perinatal period. Annu Rev Physiol 1995;57:115-34.

14. Teitel DF, Iwamoto HS, Rudolph AM. Changes in the pulmonary circulation during birth-related events. Pediatr Res 1990;27(4 Pt 1):372-8.

15. Hooper SB. Role of luminal volume changes in the increase in pulmonary blood flow at birth in sheep. Exp Physiol 1998;83:833-42.

16. Moore P, Velvis H, Fineman JR, Soifer SJ, Heymann MA. EDRF inhibition attenuates the increase in pulmonary blood flow due to oxygen ventilation in fetal lambs. J Appl Physiol 1992;73:2151-7.

17. Dawes GS. Fetal and Neonatal Physiology. Chicago, IL: Year Book, 1968.

18. Fuhrman BP, Everitt J, Lock JE. Cardiopulmonary effects of unilateral airway pressure changes in intact infant lambs. J Appl Physiol Respir Environ Exerc Physiol 1984;56:1439-48.

19. Fuhrman BP, Smith-Wright DL, Kulik TJ, Lock JE. Effects of static and fluctuating airway pressure on intact pulmonary circulation. J Appl Physiol 1986;60:114-22.

20. Lang JA, Pearson JT, te Pas AB, et al. Ventilation/perfusion mismatch during lung aeration at birth. J Appl Physiol 2014;117:535-43.

21. Seed M, van Amerom JF, Yoo SJ, et al. Feasibility of quantification of the distribution of blood flow in the normal human fetal circulation using CMR: a cross-sectional study. J Cardiovasc Magn Reson 2012;14:79.

22. Polglase GR, Wallace MJ, Grant DA, Hooper SB. Influence of fetal breathing movements on pulmonary hemodynamics in fetal sheep. Pediatr Res 2004;56:932-8.

23. Crossley KJ, Allison BJ, Polglase GR, Morley CJ, Davis PG, Hooper SB. Dynamic changes in the direction of blood flow through the ductus arteriosus at birth. J Physiol 2009;587(Pt 19):4695-704.
24. Grant DA, Hollander E, Skuza EM, Fauchère JC. Interactions between the right ventricle and pulmonary vasculature in the fetus. J Appl Physiol 1999;87:1637-43.

25. Bhatt $\mathrm{S}$, Alison BJ, Wallace EM, et al. Delaying cord clamping until ventilation onset improves cardiovascular function at birth in preterm lambs. J Physiol 2013;591(Pt 8):2113-26.

26. Niermeyer S, Velaphi S. Promoting physiologic transition at birth: reexamining resuscitation and the timing of cord clamping. Semin Fetal Neonatal Med 2013;18:385-92.

27. Begley CM, Gyte GML, Devane D, McGuire W, Weeks A. Active versus expectant management for women in the third stage of labour. Cochrane Database of Systematic Reviews 2011;11:CD007412.

28. Huh WK, Chelmow D, Malone FD. A double-blinded, randomized controlled trial of oxytocin at the beginning versus the end of the third stage of labor for prevention of postpartum hemorrhage. Gynecol Obstet Invest 2004;58:72-6.

29. Jackson KW Jr, Allbert JR, Schemmer GK, Elliot M, Humphrey A, Taylor J. A randomized controlled trial comparing oxytocin administration before and after placental delivery in the prevention of postpartum hemorrhage. Am J Obstet Gynecol 2001;185:873-7.

30. Yao AC, Hirvensalo M, Lind J. Placental transfusion-rate and uterine contraction. Lancet 1968;1:380-3.

31. McDonald SJ, Middleton P, Dowswell T, Morris PS. Effect of timing of umbilical cord clamping of term infants on maternal and neonatal outcomes. Cochrane Database Syst Rev 2013;7:CD004074.

32. Perlman JM, Wyllie J, Kattwinkel J, et al. Neonatal resuscitation: 2010 international consensus on cardiopulmonary resuscitation and emergency cardiovascular care science with treatment recommendations. Pediatr 2010;126:e1319-44.

33. Roehr CC, Hansmann G, Hoehn T, Bührer C. The 2010 Guidelines on Neonatal Resuscitation (AHA, ERC, ILCOR): similarities and differenceswhat progress has been made since 2005? Klin Padiatr 2011;223:299-307.

34. Kitchen MJ, Paganin D, Lewis RA, Yagi N, Uesugi K. Analysis of speckle patterns in phase-contrast images of lung tissue. Nucl Instru Methods Phys Res 2005;548:240-6. 